\title{
UNCONDITIONAL BASES AND STRICTLY CONVEX DUAL RENORMINGS
}

\author{
R. J. SMITH AND S. TROYANSKI
}

\begin{abstract}
We present equivalent conditions for a space $X$ with an unconditional basis to admit an equivalent norm with a strictly convex dual norm.
\end{abstract}

\section{INTRODUCTION}

We say that a norm $\|\cdot\|$ on a Banach space $X$ is strictly convex, or rotund, if $x=y$ whenever $\|x\|=\|y\|=\frac{1}{2}\|x+y\|$. Geometrically, $\|\cdot\|$ is strictly convex if its unit sphere contains no non-trivial line segments. The norms of many classical spaces fail to possess this property, however, it is often possible to introduce a new, equivalent norm that does. Therefore we seek verifiable conditions that allow us to determine when such a renorming is possible.

While the notion of strict convexity has been established now for several decades, it has eluded general characterisation. In this note, we study the class of spaces $X$ with an unconditional basis (generally uncountable). In section 2, we obtain equivalent conditions for $X$ to admit an equivalent norm, such that its dual norm is strictly convex. The tools used are topological.

It should be noted that, in the context of spaces with unconditional bases, equivalent conditions for the existence of some other types of norm are known. We say that $\|\cdot\|$ is locally uniformly rotund, or $L U R$, if, given $x$ and $x_{n}$ in $X$ such that $\|x\|=\left\|x_{n}\right\|=1$ and $\left\|x+x_{n}\right\| \rightarrow 2$, we have $\left\|x-x_{n}\right\| \rightarrow 0$. Clearly, if $\|\cdot\|$ is LUR then it is also strictly convex. The norm $\|\cdot\|$ is said to be Gâteaux smooth if, given non-zero $x$, we have

$$
\lim _{t \rightarrow 0} \frac{\|x+t h\|+\|x-t h\|-2\|x\|}{t}=0 .
$$

If, for all non-zero $x$, this limit exists uniformly for $h$ in the unit sphere of $X$, then $\|\cdot\|$ is Fréchet smooth. By a well known result of Šmulyan (cf [5, Theorem I.1.4]), if the dual norm of $\|\cdot\|$ is strictly convex (respectively LUR) then $\|\cdot\|$ is Gâteaux (respectively Fréchet) smooth. The converses do not hold, even up to renormings. In fact, there exists a space with a Fréchet smooth norm, which does not admit any equivalent norm with a strictly convex dual norm (cf [5, Theorem VII.5.2 (ii)]).

Date: November 2008.

2000 Mathematics Subject Classification. 46B03; 46B26; 46B15.

Key words and phrases. Strictly convex, norm, unconditional basis.

Supported by MTM 2008-05396/MTM/Fondos FEDER and Fundatión Séneca 0690/PI/04 CARM and Institute of Mathematics and Informatics Bulgarian Academy of Sciences, and grant MM-1401/2004 of Bulgarian NSF. 
However, in the class of spaces with unconditional bases, we do have equivalence up to a renorming.

Theorem 1.1. Let $X$ have an unconditional basis. Then $X$ admits an equivalent norm with LUR dual norm whenever $X$ admits an equivalent Fréchet smooth norm.

This result has been known since the 1960s. Indeed, in [18, the second named author proved that if $X$ has a unconditional basis then $X$ admits an equivalent LUR norm. Since [18 is written in Russian and is of limited availability, for convenience, we define this LUR norm here. Let $\|\cdot\|$ be the original norm on $X$ and $\left(e_{\gamma}\right)_{\gamma \in \Gamma}$ an unconditional basis with conjugate system $\left(f_{\gamma}\right)_{\gamma \in \Gamma}$. By a renorming, we may assume that $\left(e_{\gamma}\right)_{\gamma \in \Gamma}$ is 1-unconditional with respect to $\|\cdot\|$. Recall Day's norm $\|\cdot\|_{\text {Day }}$ on $\ell_{\infty}(\Gamma)$ (cf [5, Definition II.7.2]) and the fact that $\|\cdot\|_{\text {Day }}$ is LUR when restricted to $c_{0}(\Gamma)\left(\operatorname{cf}\left[5\right.\right.$, Theorem II.7.3]). Define $T: X \longrightarrow c_{0}(\Gamma)$ by $(T x)(\gamma)=f_{\gamma}(x)$ for $x \in X$ and $\gamma \in \Gamma$, and set

$$
\|\| x\left\|^{2}=\right\| T x\left\|_{\text {Day }}^{2}+\sum_{n=0}^{\infty} 2^{-n}\right\| x \|_{n}^{2}
$$

where

$$
\|x\|_{n}^{2}=\sup \left\{\left\|\sum_{\gamma \in \Gamma \backslash A} f_{\gamma}(x) e_{\gamma}\right\|+2 \sum_{\gamma \in A}\left|f_{\gamma}(x)\right|: A \subseteq \Gamma \text { and } \operatorname{card} A \leq n\right\} .
$$

Then $\||\cdot|\|$ is LUR.

Proof of Theorem 1.1. Let $X$ have a 1-unconditional basis $\left(e_{\gamma}\right)_{\gamma \in \Gamma}$, with conjugate system $\left(f_{\gamma}\right)_{\gamma \in \Gamma}$. If $X$ admits an equivalent Fréchet smooth norm then it cannot contain an isomorphic copy of $\ell_{1}$, for $\ell_{1}$ admits no such norm (cf [5, Corollary II.3.3]). Hence $\left(e_{\gamma}\right)_{\gamma \in \Gamma}$ is shrinking (cf [10, Theorem 1.c.9]), and it follows that the conjugate system $\left(f_{\gamma}\right)_{\gamma \in \Gamma}$ is an unconditional basis of $X^{*}$. Now it is a straightforward matter to verify that the LUR norm $\||\cdot|\|$ defined above, but on $X^{*}$, is $w^{*}$-lower semicontinuous and thus the dual of an equivalent norm on $X$.

In particular, if $X$ has an unconditional basis then $X$ admits an equivalent norm with a LUR dual norm if and only if $X$ does not cannot contain any isomorphic copies of $\ell_{1}$. In the dual strictly convex case, $X$ may contain copies of $\ell_{1}$ but, very roughly speaking, it cannot contain too many of them. We make this statement clearer in Remark 2.10, Note also that if $\Gamma$ is uncountable and $X$ contains an isomorphic copy of $\ell_{1}(\Gamma)$, then it is known that $X$ cannot admit any equivalent norm with a strictly convex dual norm. Indeed, $\ell_{1}(\Gamma)$ does not even admit an equivalent Gâteaux smooth norm [4, Theorem 9].

In Section 2, we present our main topological tools, Lemma 2.1 and Theorem 2.5, together with Theorem 2.9, our characterisation. In section 3, we apply the topological tools to examples from the literature. 


\section{Strictly CONVEX DUAL NORMS}

Let $\Gamma$ be a set, and suppose that the real Banach space $X$ has a normalised unconditional basis $\left(e_{\gamma}\right)_{\gamma \in \Gamma}$. We shall identify both $X$ and $X^{*}$ as vector sublattices of $\mathbb{R}^{\Gamma}$ in the natural way, with the pointwise lattice order. Moreover, both sublattices are ideals, in the sense that if $x \in \mathbb{R}^{\Gamma}, y \in X$ and $|x| \leq y$, then $x \in X$, and similarly for $X^{*}$. By a renorming, we can assume that the basis is 1-unconditional, which means that $X$ and $X^{*}$ are both Banach lattices. It will be helpful to keep the lattice structure of $X$ and $X^{*}$ in mind. It is clear that the dual norm of $X^{*}$ is always finer than $\|\cdot\|_{\infty}$, which we define on $X^{*}$ in the obvious way.

We must define some topological concepts. A function $d: Z \times Z \longrightarrow[0, \infty)$ is called a symmetric if it satisfies all the axioms for a metric, with the possible exception of the triangle inequality. Let $(Z, \tau)$ be a regular topological space. We say that $Z$ is fragmentable if there exists a metric $d$ on $Z$ with the property that, for any non-empty subset $E \subseteq Z$ and $\varepsilon>0$, there exists a $\tau$-open set $U$ such that $E \cap U$ is non-empty and has $d$-diameter not exceeding $\varepsilon$. In fact, by [15, Remark 1.10], for $Z$ to be fragmentable, we only require that $d$ is a non-negative function on $Z \times Z$, with the property that $x=y$ whenever $d(x, y)=0$. A family of subsets $\mathscr{N}$ of $Z$ is a network for $Z$ if, given $x \in U \in \tau$, there exists $N \in \mathscr{N}$ such that $x \in N \subseteq U$. A family of subsets $\mathscr{F}$ is called isolated if $E \cap \overline{U \mathscr{F} \backslash\{E\}}$ is empty whenever $E \in \mathscr{F}$; equivalently, there is some $U \in \tau$ such that $E \subseteq U$ and $U \cap F$ is empty for all $F \in \mathscr{F} \backslash\{E\}$. A network $\mathscr{N}$ is called $\sigma$-isolated if it can be written as $\mathscr{N}=\bigcup_{n=1}^{\infty} \mathscr{N}_{n}$, where each $\mathscr{N}_{n}$ is isolated. We will say that $Z$ is descriptive if it is compact and admits a $\sigma$-isolated network. The class of descriptive compact spaces is broad and encompasses all metrisable compacta, as well as Eberlein and Gul'ko compacta, which we shall consider later, in Section 3. Symmetrics, $\sigma$-isolated networks and fragmentable and descriptive spaces have been studied in, for example, [7, 9, 14, 15]. We say that $Z$ is a Gruenhage space if there exist families $\left(\mathscr{U}_{n}\right)_{n \in \mathbb{N}}$ of open sets with the property that given distinct $x, y \in Z$, there exist $n \in \mathbb{N}$ and $U \in \mathscr{U}_{n}$ such that $U \cap\{x, y\}$ is a singleton, and either $x$ lies in finitely many $U^{\prime} \in \mathscr{U}_{n}$, or $y$ lies in finitely many $U^{\prime} \in \mathscr{U}_{n}$. Gruenhage spaces were introduced in [8] and have recently found application in the theory of strictly convex dual norms [16]. Every descriptive compact space is Gruenhage. We let $\tau_{p}$ denote the pointwise topology on $\mathbb{R}^{\Gamma}$. We will introduce further classes of compact topological spaces in Section 3 .

Lemma 2.1. Let $K \subseteq[0,1]^{\Gamma}$ be $\tau_{p}$-compact satisfy $x \wedge y \in K$ whenever $x, y \in K$. Suppose further that there exists a $\tau_{p}$-lower semicontinuous function $\rho: K \longrightarrow[0,1]$ such that

$(*)$ if $y<x$ then there exists $\alpha<\rho(x)$ and an open set $U \ni y$ with the property that if $z \leq x$ and $z \in U$, then $\rho(z)<\alpha$.

Then we deduce the following

I. $K$ is fragmentable;

II. for all $r \in[0,1],\left(\rho^{-1}(r), \tau_{p}\right)$ has a $\sigma$-isolated network.

Moreover, if $K \subseteq\{0,1\}^{\Gamma}$, then

III. $K$ is descriptive. 
Proof. (I). For $x, y \in K$, set $d(x, y)=\max \{\rho(x), \rho(y)\}-\rho(x \wedge y)$. Note that (*) implies that $\rho$ is strictly increasing, that is, $\rho(y)<\rho(x)$ whenever $y<x$. Thus, if $d(x, y)=0$ then $x=x \wedge y=y$, so $d$ is a symmetric. We show that it fragments $K$. Indeed, if $M \subseteq K$ is non-empty and $\varepsilon>0$, let $\alpha=\sup _{x \in M} \rho(x)$. Take $x \in M$ such that $\rho(x)>\alpha-\varepsilon$. By the lower semicontinuity, there exists an open set $U \ni x$ such that $\rho(y)>\rho(x)-\varepsilon$ whenever $y \in U$. Moreover, we can assume that $y \wedge z \in U$ whenever $y, z \in U$. In particular, if $y, z \in M \cap U$ then $d(y, z) \leq \alpha-\rho(y \wedge z)<\alpha-(\alpha-2 \varepsilon)=2 \varepsilon$. By [15, Remark 1.10] mentioned in the preamble to this section, $K$ is fragmentable.

(II). For $x \in K$ and $\varepsilon>0$, define

$$
B(x, \varepsilon)=\{y \in K: \rho(y) \leq \rho(x) \text { and } d(x, y)<\varepsilon\} .
$$

Since $y \mapsto \rho(x \wedge y)$ is $\tau_{p}$-lower semicontinuous, $B(x, \varepsilon)$ is open in $\rho^{-1}[0, \rho(x)]$. We prove that if the sequence $\left(x_{n}\right)$ satisfies $\max \left\{\rho(x), \rho\left(x_{n}\right)\right\} \rightarrow \rho(x)$ and $d\left(x, x_{n}\right) \rightarrow 0$, then $x_{n} \rightarrow x$. Indeed, first suppose that $\left(x_{n_{r}}\right)$ is a subsequence converging to some $y \in K$. We have $\rho(y) \leq \liminf \rho\left(x_{n_{r}}\right) \leq \rho(x)$. We claim that $x \leq y$. For a contradiction, suppose otherwise. Since $x_{n_{r}} \rightarrow y$, we have $x \wedge x_{n_{r}} \rightarrow x \wedge y<x$. Thus by (*), there exists $\alpha<\rho(x)$ and an open set $U \ni x \wedge y$ such that if $z \leq x$ and $z \in U$ then $\rho(z)<\alpha$. It follows that

$$
\begin{aligned}
d\left(x, x_{n_{r}}\right) & =\max \left\{\rho(x), \rho\left(x_{n_{r}}\right)\right\}-\rho\left(x \wedge x_{n_{r}}\right) \\
& \geq \rho(x)-\rho\left(x \wedge x_{n_{r}}\right) \\
& >\rho(x)-\alpha \\
& >0
\end{aligned}
$$

for large enough $r$, which is a contradiction. This proves our claim that $x \leq y$, and since $\rho(y) \leq \rho(x)$ and $\rho$ is strictly increasing, we conclude that $y=x$. Being fragmentable and compact, $K$ is also sequentially compact, so $x_{n} \rightarrow x$. Thus, if $x \in U \subseteq \rho^{-1}(r)$, with $U$ open in $\rho^{-1}(r)$, then there exists $\varepsilon>0$ such that $B(x, \varepsilon) \cap \rho^{-1}(r) \subseteq U$. Consequently, $d$ semi-metrises $\rho^{-1}(r)$ for every $r$. Since $K$ is compact, it is fragmentable by a metric which generates a finer topology than $\tau_{p}$ [15, Corollary 1.11], and thus each $\rho^{-1}(r)$ is so fragmented. It follows that $\rho^{-1}(r)$ has a $\sigma$-isolated network by [7, Theorems 9.8 and 5.11] and [14, Lemma 2.2].

(III). Suppose now that $K \subseteq\{0,1\}^{\Gamma}$. We can and do assume that $\rho\left(K \backslash\left\{\chi_{\varnothing}\right\}\right) \subseteq$ $\left[\frac{1}{2}, 1\right]$. If we define

$$
M=\left\{\lambda \chi_{A}: \chi_{A} \in K, \lambda \in[0,2]\right\},
$$

$\sigma\left(\lambda \chi_{A}\right)=\lambda \rho(A)$ for $A \neq \varnothing, \lambda>0$, and $\sigma\left(\chi_{\varnothing}\right)=0$, it is straightforward to verify that $M$ is $\tau_{p}$-compact and $\sigma$ is a $\tau_{p}$-lower semicontinuous function on $M$. It is clear that $x \wedge y \in M$ whenever $x, y \in M$. To see that $\sigma$ satisfies $(*)$ too, we take $x=\lambda \chi_{A}, y=\mu \chi_{B} \in M$ such that $y<x$. Clearly $A$ is non-empty and $\lambda>0$. In addition, we can assume that either (a) $A=B$ and $0<\mu<\lambda$, or (b) $B \varsubsetneqq A$ and $\mu \leq \lambda$. If (a) holds then put $\beta=\frac{1}{2}(\lambda+\mu) \rho\left(\chi_{A}\right)<\sigma(x)$, select $\gamma \in A$ and define the open set $V=\left\{z \in M: 0<z_{\gamma}<\frac{1}{2}(\lambda+\mu)\right\}$. If $z=\nu \chi_{C} \leq x$ and $z \in V$ then $C \subseteq A$ and $\nu<\frac{1}{2}(\lambda+\mu)$, giving $\sigma(z)<\frac{1}{2}(\lambda+\mu) \rho\left(\chi_{A}\right)=\beta$. If (b) holds then take $\alpha<\rho\left(\chi_{A}\right)$ and an open neighbourhood $U \subseteq K$ of $\chi_{B}$ with the property 
that $\rho\left(\chi_{C}\right)<\alpha$ whenever $\chi_{C} \in U$. Let $\beta=\frac{\lambda}{2}\left(\alpha+\rho\left(\chi_{A}\right)\right)<\sigma(x)$, and observe that $V=\left\{\nu \chi_{C}: \chi_{C} \in U, \nu<\beta / \alpha\right\}$ is open in $M$. Moreover, $y \in V$ and, if $z=\nu \chi_{C} \leq x$ and $z \in V$ then $\sigma(z)<\beta$.

By (II), $\left(S, \tau_{p}\right)$ has a $\sigma$-isolated network, where $S=\sigma^{-1}(1)$. Following [9, Theorem 7.2], $\left(L, \tau_{p}\right)$ also has a $\sigma$-isolated network, where $L=\sigma^{-1}[0,1]$. We sketch an argument for completeness. The map $(t, x) \mapsto(t, t x)$ is a homeomorphism of $(0,1] \times S$ and a subset $E$ of $(0,1] \times L \backslash\{0\}$, so $E$ has a $\sigma$-isolated network. If $y=\lambda_{\chi_{A}} \in L \backslash\{0\}$ then $x=\frac{1}{\sigma(y)} y=\frac{1}{\rho\left(\chi_{A}\right)} \chi_{A}$ is an element of $M$ because $\rho\left(\chi_{A}\right) \geq \frac{1}{2}$. Moreover $x \in S$ and $(\sigma(y), y) \in E$. Thus $E$ projects onto $L \backslash\{0\}$, and so $L \backslash\{0\}$ has a $\sigma$-isolated network, again by the proof of [9, Theorem 7.2]. To finish, we simply adjoin $\{0\}$ to the network. Since $K$ embeds in $L$, we have proved (III).

Lemma 2.1 will be applied first to a specific class of topological spaces and an associated class of Banach spaces.

Definition 2.2. A family of subsets $\mathscr{A}$ of $\Gamma$ is called adequate on $\Gamma$, or simply adequate, if it satisfies the following three conditions:

(1) $\{\gamma\} \in \mathscr{A}$ for all $\gamma \in \Gamma$;

(2) $B \in \mathscr{A}$ whenever $A \in \mathscr{A}$ and $B \subseteq A$;

(3) if every finite subset of $A \subseteq \Gamma$ is in $\mathscr{A}$ then $A \in \mathscr{A}$.

Note that we can replace property (3) of Definition 2.2 with

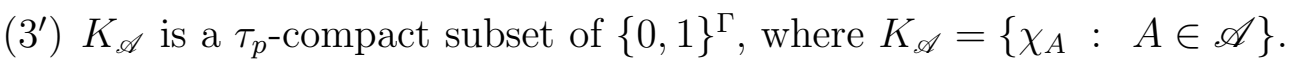

The set of totally ordered subsets of a partially ordered set $E$ is adequate on $E$. If $\Gamma$ is a set of consistent sentences in a first-order theory then the family of consistent subsets of $\Gamma$ is adequate on $\Gamma$. Adequate families were defined in [17] and have been considered subsequently by several authors in, for example, [3, 11]. Given an adequate family $\mathscr{A}$, we define Banach lattice ideal $\ell_{\mathscr{A}}$ as the set of all $x \in \ell_{\infty}(\Gamma)$ satisfying $\|x\|_{\mathscr{A}}<\infty$, where $\|x\|_{\mathscr{A}}=\sup _{A \in \mathscr{A}}\left\|x \uparrow_{A}\right\|_{1}$, where $\|\cdot\|_{1}$ is the standard 1-norm (cf [3, Definition 2.1]). For example, if $\mathscr{A}=\{\varnothing\} \cup\{\{\gamma\}: \gamma \in \Gamma\}$ then $\ell_{\mathscr{A}}=\ell_{\infty}(\Gamma)$, and if $\Gamma \in \mathscr{A}$ then $\ell_{\mathscr{A}}=\ell_{1}(\Gamma)$. It is easy to see that, in general, the standard unit vectors $\left(e_{\gamma}\right)_{\gamma \in \Gamma}$ form a normalised 1-unconditional basic sequence in $\ell_{\mathscr{A}}$. We set $h_{\mathscr{A}}=\overline{\operatorname{span}}\|\cdot\|_{\mathscr{A}}\left(e_{\gamma}\right)_{\gamma \in \Gamma}$ and denote the dual norm on $h_{\mathscr{A}}^{*}$ again by $\|\cdot\|_{\mathscr{A}}$. Given $x \in h_{\mathscr{A}}$ and $A \subseteq \Gamma$, we define

$$
\mathbf{1}_{A}(x)=\sum_{\gamma \in A} x_{\gamma}
$$

whenever this sum makes sense. It is clear that the functions $\mathbf{1}_{A}, A \in \mathscr{A}$, lie in $h_{\mathscr{A}}^{*}$, with $\left\|\mathbf{1}_{A}\right\|_{\mathscr{A}}=1$ whenever $A$ is non-empty. It is also easy to verify that the map $\pi: \chi_{A} \mapsto \mathbf{1}_{A}$ on $K_{\mathscr{A}}$ is $\tau_{p}-w^{*}$ continuous; in particular, the image $\pi\left(K_{\mathscr{A}}\right)$ is homeomorphic to $K_{\mathscr{A}}$. The proof of Theorem 2.5 below requires some renorming results, which we state partially.

Theorem 2.3 ([16]).

(1) Let $K$ be a Gruenhage compact space. Then $\mathscr{C}(K)$ admits a norm with a strictly convex dual norm. 
(2) Let $\left(B_{X^{*}}, w^{*}\right)$ be a Gruenhage compact space. Then $X$ admits a norm with a strictly convex dual norm.

Theorem $2.4\left(\left[12\right.\right.$, Theorem 2.6]). Let $(X,\|\cdot\|)$ be a Banach lattice ideal of $\mathbb{R}^{\Gamma}$, such that $\|\cdot\|_{\infty} \leq\|\cdot\|$. Then $X$ admits a $\tau_{p}$-lower semicontinuous, strictly convex norm if and only if $X$ admits a $\tau_{p}$-lower semicontinuous norm $\||\cdot|||$ satisfying $\||| x|\|<\|||y|\|$ whenever $|x|<|y|$.

Theorem 2.5. Let $\mathscr{A}$ be an adequate family. Then the following are equivalent.

(1) $K_{\mathscr{A}}$ is a Gruenhage compact;

(2) $h_{\mathscr{A}}$ admits a norm with strictly convex dual norm;

(3) there exists a strictly increasing, $\tau_{p}$-lower semicontinuous map $\rho: K_{\mathscr{A}} \longrightarrow$ $[0,1]$;

(4) $K_{\mathscr{A}}$ is a descriptive compact.

Proof. (1) $\Rightarrow(2)$. If $\mathscr{A}$ is a Gruenhage compact then $\mathscr{C}(\mathscr{A})$ admits an equivalent norm $\|\cdot\|$ with a strictly convex dual norm by Theorem [2.3, part (1). Define $T$ : $h_{\mathscr{A}} \longrightarrow \mathscr{C}(\mathscr{A})$ by $(T x)(A)=\chi_{A}(x)$ and observe that, since $\mathscr{A}$ is adequate, we have $\frac{1}{2}\|x\|_{\mathscr{A}} \leq\|T x\|_{\infty} \leq\|x\|_{\mathscr{A}}$. Consequently, the dual norm of $\|\cdot \cdot\|$, where \|\|$x\|=\| T x \|, x \in h_{\mathscr{A}}$, is strictly convex. (2) $\Rightarrow(3)$ follows from Theorem 2.4 and the natural embedding of $K_{\mathscr{A}}$ in $B_{h_{\mathscr{A}}^{*}}$ defined above. (3) $\Rightarrow(4)$. If $\chi_{B}<\chi_{A}$ then take $\gamma \in A \backslash B$. Property ( $*$ ) of Lemma 2.1 is fulfilled by setting $\alpha=\rho(A \backslash\{\gamma\})$ and $U=\left\{\chi_{C}: \gamma \notin C\right\} .(4) \Rightarrow(1)$ follows from [16, Corollary 4].

Remark 2.6. The proof of the implication $(1) \Rightarrow(4)$ in Theorem 2.5 uses a renorming of a Banach space. The authors would be interested to see a direct, purely topological proof of this result. The proof of Theorem 2.5 also shows that if $\mathscr{A}$ is adequate then $\mathscr{C}\left(K_{\mathscr{A}}\right)$ admits a norm with strictly convex dual norm if and only if $K_{\mathscr{A}}$ is a Gruenhage space. We don't know if the direct implication holds in general.

We finish this section by providing a characterisation of spaces with unconditional bases which admit an equivalent norm with strictly convex dual norm. Let $X$ have a normalised, 1-unconditional basis $\left(e_{\gamma}\right)_{\gamma \in \Gamma}$. Let $\|\cdot\|$ denote the dual norm on $X^{*}$. Define

$$
\mathscr{A}=\left\{A \subseteq \Gamma: \mathbf{1}_{A} \in X^{*}\right\} .
$$

The family $\mathscr{A}$ contains all singletons $\{\gamma\}, \gamma \in \Gamma$, and is closed under taking subsets and finite unions. Hence $\mathscr{A}$ is adequate if and only if $X$ is isomorphic to $\ell_{1}(\Gamma)$. While $K_{\mathscr{A}}=\left\{\chi_{A}: A \in \mathscr{A}\right\}$ is not compact in general, it is $\sigma$-compact because $K_{A}=\bigcup_{n=1}^{\infty} K_{\mathscr{A}_{n}}$, where $\mathscr{A}_{n}$ is the adequate family

$$
\left\{A \in \mathscr{A}:\left\|\mathbf{1}_{A}\right\| \leq n\right\} .
$$

Let $W$ be the linear span of

$$
\left\{\mathbf{1}_{A}: A \in \mathscr{A}, n \in \mathbb{N}\right\} .
$$

While it is not true that $\bar{W}^{\|\cdot\|}=X^{*}$ in general, it is clear that $\bar{W}^{\|\cdot\|_{\infty}}=X^{*}$. We require the following result. 
Proposition 2.7 ([16, Corollary 10]). Let $X$ be a Banach space and suppose that $X^{*}=\operatorname{span}^{|| \cdot|| \mid}(K)$, where $K$ is a Gruenhage compact in the $w^{*}$-topology and $\||\cdot|\|$ is equivalent to a coarser, $w^{*}$-lower semicontinuous norm on $X^{*}$. Then $X^{*}$ admits an equivalent, strictly convex dual norm.

A norm on a dual space $X^{*}$ is said to be $w^{*}-L U R$ if, given $x$ and $x_{n}$ in $X^{*}$ such that $\|x\|=\left\|x_{n}\right\|=1$ and $\left\|x+x_{n}\right\| \rightarrow 2$, we have $x_{n} \rightarrow x$ in the $w^{*}$ topology. Such norms are studied in [14].

Theorem 2.8 ([14, Theorem 1.3]). If $B_{X^{*}}$ is a descriptive compact space in the $w^{*}$-topology then $X$ admits an equivalent norm with $w^{*}-L U R$ dual norm.

The next result is our promised characterisation.

Theorem 2.9. Let $X$ have a normalised 1-unconditional basis, with $\mathscr{A}_{n}, n \in \mathbb{N}$, defined as above. Then the following are equivalent.

(1) $\left(B_{X^{*}}, w^{*}\right)$ is a Gruenhage compact space;

(2) $X$ admits an equivalent norm with a strictly convex dual norm;

(3) there exists a strictly increasing, $\tau_{p}$-lower semicontinuous map

$$
\rho: K_{\mathscr{A}} \longrightarrow[0,1]
$$

(4) $\left(B_{X^{*}}, w^{*}\right)$ is a descriptive compact space;

(5) $X$ admits an equivalent norm with a $w^{*}-L U R$ dual norm.

Proof. (1) $\Rightarrow$ (2) follows from Theorem 2.3, part (2). (4) $\Rightarrow(5)$ follows from Theorem 2.8. $(5) \Rightarrow(2)$ is an immediate consequence of the definition and $(4) \Rightarrow(1)$ is [16. Corollary 4]. We prove $(2) \Leftrightarrow(3)$ and $(2) \Rightarrow(4)$. Suppose that (2) holds. Using Theorem 2.4, we can find a strict lattice dual norm \|\|$\cdot\|\|$ on $X^{*}$. It is easy to see that the map $\chi_{A} \mapsto\left\|\mathbf{1}_{A}\right\|||$ satisfies (4). Now suppose that $\rho$ satisfies (3). Let $K_{n}=\left\{\mathbf{1}_{A}: A \in \mathscr{A}_{n}\right\}$, where $\mathscr{A}_{n}$ is as above. By Theorem [2.5, each $K_{n}$ is a descriptive compact in the $w^{*}$-topology. If we set $K=\bigcup_{n=1}^{\infty} n^{-2} K_{n} \cup\{0\}$ then $K$ is again descriptive, and $\overline{\operatorname{span}}\|\cdot\|_{\infty}(K)=\bar{W}^{\|\cdot\|_{\infty}}=X^{*}$, where $W$ is as above. Since $\|\cdot\|_{\infty}$ is a $w^{*}$-lower semicontinuous norm on $X^{*}$, coarser than $\|\cdot\|$, we can apply Proposition 2.7 to obtain an equivalent, strictly convex dual norm on $X^{*}$.

To finish, we prove $(2) \Rightarrow(4)$. Given (2), let $\||\cdot|\| \mid$ be as above, and identify the

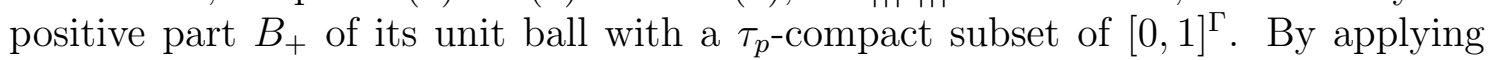
Lemma 2.1 to $B_{+}$with $\rho=\|\| \cdot\|\|$, we have that $\left\{f \in B_{+}:\|\| f\|\|=1\right\}$ has a $\sigma$ isolated network in the $\tau_{p}$ (equivalently $w^{*}$ ) topology. It follows from [9, Theorem $7.2]$ that $\left(B_{+}, w^{*}\right)$ has a $\sigma$-isolated network, so is a descriptive compact. Because descriptive compact spaces are preserved under continuous images [13, Corollary 3.4], we have that $B \subseteq B_{+}-B_{+}$is descriptive.

Remark 2.10. We observe that $A \in \mathscr{A}$ if and only if $\left(x_{\gamma}\right)_{\gamma \in A} \mapsto \sum_{\gamma \in A} x_{\gamma} e_{\gamma}$ defines an isomorphism from $\ell_{1}(A)$ into $X$. Thus Theorem 2.9, part (3), is a more precise formulation of the assertion, made after the proof of Theorem 1.1, that in the dual strictly convex case $X$ cannot contain too many copies of $\ell_{1}$. 
Finally, we note that there is a Banach space of type $h_{\mathscr{A}}$ which does not satisfy the conditions of Theorems 2.5] or 2.9] and does not contain an isomorphic copy of $\ell_{1}(\Gamma)$ for any uncountable $\Gamma$. See [3, Theorems 1.7 and $\left.3.6(\mathrm{c})\right]$.

\section{EXAMPLES}

In this section, we apply Lemma 2.1 and Theorem 2.5 to obtain some new results concerning examples of compact spaces from the literature.

Definition 3.1. We shall say that a partially ordered set $(T,<)$ is a pseudotree (respectively tree) if, for every $x \in T$, the set $I_{x}=\{w \in T: w<x\}$ is totally (respectively well) ordered.

Pseudotrees were introduced by Kurepa and studied in [11] under the name of bushes. We say that a subset $\Gamma$ of a partially ordered set is an antichain if no two distinct elements of $\Gamma$ are comparable. A partially ordered set is called special if it can be written as a countable union of antichains. Given a pseudotree $T$, we let $\mathscr{A}_{T}$ be the adequate family of all totally ordered subsets of $T$. Such families were investigated in the context of Talagrand compact spaces in [11]. A compact space $K$ is called Talagrand if the Banach space $\mathscr{C}(K)$ is $\mathcal{K}$-analytic in its weak topology; see, for example, [1, 11, 17] for details.

Proposition 3.2 ([11, Theorem 3.2]). Let $T$ be a pseudotree. Then $K_{\mathscr{A}_{T}}$ is a Talagrand compact if and only if $T$ is a countable union of antichains.

We can use Theorem 2.5 to provide a straightforward extension of this result. Recall that a compact space $K$ is called Eberlein if it is homeomorphic to a weakly compact subset of a Banach space. The implications Eberlein $\Rightarrow$ Talagrand $\Rightarrow$ descriptive $\Rightarrow$ Gruenhage have been established and are known to be strict.

Proposition 3.3. Let $T$ be a pseudotree. Then the following are equivalent.

(1) $K_{\mathscr{A}_{T}}$ is Eberlein;

(2) $K_{\mathscr{A}_{T}}$ is Gruenhage;

(3) $T$ is special.

Proof. Only $(2) \Rightarrow(3)$ and $(3) \Rightarrow(1)$ require proof. Assume (2). By Theorem 2.5, there exists a strictly increasing map $\rho: K_{\mathscr{A}_{T}} \longrightarrow[0,1]$. If we pick $\sigma(x) \in$ $\left(\rho\left(\chi_{I_{x}}\right), \rho\left(\chi_{I_{x} \cup\{x\}}\right)\right) \cap \mathbb{Q}$ for each $x \in T$, it is evident that $\sigma: T \longrightarrow \mathbb{Q}$ is strictly increasing, and that the fibres $\sigma^{-1}(q), q \in \mathbb{Q}$, are antichains.

Assume (3). Let $T=\bigcup_{n \in \mathbb{N}} \Gamma_{n}$, where $\Gamma_{n}, n \in \mathbb{N}$, is a pairwise disjoint family of antichains. It is clear that the map $\pi: K_{\mathscr{A}_{T}} \longrightarrow c_{0}(T)$, defined by $\pi\left(\chi_{A}\right)(x)=2^{-n}$ if $A \cap \Gamma_{n}=\{x\}$ for some $n$, and $\pi\left(\chi_{A}\right)(x)=0$ otherwise, is a homeomorphism of $\mathscr{A}_{T}$ and a weakly compact subset of $c_{0}(\Gamma)$.

Incidentally, using Proposition [3.3, we can provide more examples of spaces $h_{\mathscr{A}}$ which fail the conditions of Theorems 2.5 and 2.9 and do not contain isomorphic copies of $\ell_{1}(\Gamma)$ for any uncountable set $\Gamma$. Let $T$ be a non-special pseudotree with no uncountable branches. Plenty of such objects exist; for example, the space $\sigma \mathbb{Q}$ of well ordered subsets of $\mathbb{Q}$, partially ordered by taking initial segments, satisfies 


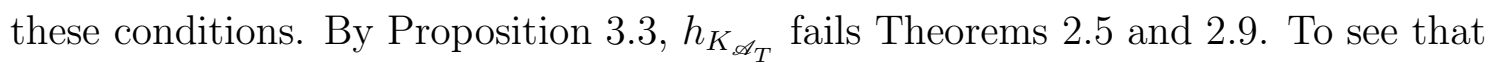
it does not contain $\ell_{1}(\Gamma)$ if $\Gamma$ is uncountable, we refer the reader to [3], Theorem 1.7 and Proposition 3.10] and [2, Theorem 3.5].

The following class of examples is based on a compact space constructed in [3]. This construction is shown to be descriptive in [13]. Here, we give an alternative proof using our strictly increasing functions.

Example 3.4 (cf [13, Example 4.5]). Let $L=\left\{(\xi, \eta) \in \omega_{1}^{2}: \xi<\eta\right\}$ and suppose that we have a function $\Phi: L \longrightarrow \mathbb{N}$. Define

$$
W=\left\{\left\{\xi_{1}, \ldots, \xi_{n}\right\} \subseteq \omega_{1}: \xi_{1}<\ldots<\xi_{n} \text { and } \Phi\left(\xi_{i}, \xi_{j}\right) \geq j \text { for all } i<j \leq n<\omega\right\}
$$

and

$$
\mathscr{A}_{\Phi}=\left\{A \subseteq \omega_{1} \text { : every finite subset of } A \text { is in } W\right\} .
$$

Then $W$ contains all singleton subsets of $\omega_{1}$ and is closed under taking subsets, and $\mathscr{A}_{\Phi}$ is adequate. We show that $K_{\mathscr{A}_{\Phi}}$ is a descriptive compact space.

Proof. If $\chi_{A} \in K_{\mathscr{A}_{\Phi}}$ then $A$ cannot have order type exceeding $\omega$. Indeed, otherwise, then we could extract elements $\xi_{1}<\ldots<\xi_{n}<\ldots<\xi_{\omega} \in A$, to give $\left\{\xi_{1}, \ldots, \xi_{n}, \xi_{\omega}\right\} \in W$ and $\Phi\left(\xi_{1}, \xi_{\omega}\right) \geq n+1$ for all $n$, which is impossible.

In order to construct a strictly increasing, $\tau_{p}$-lower semicontinuous function $\rho$ : $K_{\mathscr{A}_{\Phi}} \longrightarrow \mathbb{R}$, we first define $\pi: K_{\mathscr{A}_{\Phi}} \longrightarrow c_{0}(L)$ by

$$
\pi\left(\chi_{A}\right)(\xi, \eta)= \begin{cases}n^{-1} & \text { if } \xi, \eta \in A \text { and } \Phi(\xi, \eta)=n \\ 0 & \text { otherwise. }\end{cases}
$$

It is clear that $\pi\left(\chi_{A}\right) \in c_{0}(L)$ because there are only finitely many $(\xi, \eta) \in L \cap A^{2}$ with $\Phi(\xi, \eta)=n$. Indeed, as we have already observed, we can enumerate $A$ as a strictly increasing sequence $\left(\xi_{i}\right)_{i<\alpha}$, where $\alpha \leq \omega$. Thus, if $(\xi, \eta) \in L \cap A^{2}$ and $\Phi(\xi, \eta)=n$ then $\xi=\xi_{i}$ and $\eta=\xi_{j}$ for some $i<j$, so $\left\{\xi_{1}, \ldots, \xi_{j}\right\} \in W$ and $j \leq \Phi\left(\xi_{i}, \xi_{j}\right)=n$. Evidently, if $A \subseteq B$ then $\pi\left(\chi_{A}\right) \leq \pi\left(\chi_{B}\right)$. It is also clear that if $B$ contains at least two elements and strictly contains $A$, then $\pi\left(\chi_{A}\right)(\xi, \eta)=0<\pi\left(\chi_{B}\right)(\xi, \eta)$ for some suitable $(\xi, \eta) \in L$. Finally, we observe that $\pi$ is $\tau_{p}-\tau_{p}$ continuous.

We define our strictly increasing function $\rho$ by recalling Day's norm $\|\cdot\|_{\text {Day }}$ from the introduction and setting

$$
\rho\left(\chi_{A}\right)= \begin{cases}0 & \text { if } A=\varnothing \\ 1 & \text { if } A \text { is a singleton } \\ 1+\left\|\pi\left(\chi_{A}\right)\right\|_{\text {Day }} & \text { otherwise. }\end{cases}
$$

Since $\|\cdot\|_{\text {Day }}$ is $\tau_{p}$-lower semicontinuous and lattice, $\rho$ is $\tau_{p}$-lower semicontinuous. Being strictly convex, Day's norm is moreover strictly lattice, thus $\rho$ is strictly increasing. It follows that $K_{\mathscr{A}_{\Phi}}$ is descriptive by Theorem 2.5 .

The compact space $K$ is said to be $G u l^{\prime} k o$ if $\mathscr{C}(K)$ is $\mathcal{K}$-countably determined in its weak topology. We say that $K$ is Corson if it is homeomorphic to a subset $M$ of $[0,1]^{\Gamma}$ in the pointwise topology, with the property that the support of every element of $M$ is at most countable. See, for example, [11, 8, 2, 3, 6] for information about these classes of compact spaces. The implications Talagrand $\Rightarrow$ Gul'ko $\Rightarrow$ Corson and descriptive are known and strict. Since the order type of every $A \in \mathscr{A}_{\Phi}$ of 
Example 3.4 cannot exceed $\omega$, the associated compact space $K_{\mathscr{A}_{\Phi}}$ is always Corson. Using a particular function $\Phi$ defined in terms of a family of almost disjoint subsets of $\omega$, the authors of [3] show that the associated space $K_{\mathscr{A}_{\Phi}}$ is not Gul'ko.

Our final collection of examples is based on a class of compact spaces introduced in [1. We shall say that a subset $I$ of a pseudotree $T$ is an interval of $T$ if $s \in I$ whenever $r, t \in I$ and $r \prec s \prec t$.

Example 3.5. Let $A$ be a set and $\left(T_{a},<_{a}\right)_{a \in A}$ a family of special pseudotrees with the property that

(**) if $I$ is an interval of $T_{a}$ and $T_{b}$ for distinct $a, b \in A$, then card $I \leq 1$.

Let $T=\bigcup_{a \in A} T_{a}$ and define

$$
\Omega=\left\{\chi_{I} \in\{0,1\}^{T}: I \text { is an interval of } T_{a} \text { for some } a \in A\right\} .
$$

Using $(* *)$, it is straightforward to prove that $\Omega$ is $\tau_{p}$-compact. We show moreover that $\Omega$ is descriptive.

Proof. Since each $T_{a}$ is special, we can take a sequence $\left(A_{a, n}\right)_{n=1}^{\infty}$ of pairwise disjoint antichains of $T_{a}$ such that $T_{a}=\bigcup_{n=1}^{\infty} A_{a, n}$. If $I \in \Omega$ has at least two elements then there is a unique $a_{I} \in A$ such that $I \subseteq \Omega_{a_{I}}$. Thus we can define

$$
\rho\left(\chi_{I}\right)= \begin{cases}0 & \text { if } I=\varnothing \\ 1 & \text { if } I \text { is a singleton } \\ 1+\sum\left\{2^{-n}: I \cap A_{a_{I}, n} \neq \varnothing\right\} & \text { otherwise. }\end{cases}
$$

It is evident that $\rho$ is $\tau_{p}$-lower semicontinuous. It remains to prove that $\rho$ satisfies property (2.1) of Lemma 2.1. Suppose that $\chi_{J}<\chi_{I}$. If $I$ is the singleton $\{t\}$ then set $\alpha=\frac{1}{2}$. Otherwise $I \subseteq \Omega_{a_{I}}$. In this case take $t \in I \backslash J$ and set $\alpha=\rho\left(\chi_{I}\right)-2^{-(m+1)}$, where $m$ is the unique natural number satisfying $t \in A_{a_{I}, m}$. Note that $\alpha>1$. In both cases, define $U=\left\{\chi_{R} \in \Omega: t \notin R\right\}$. It is straightforward to verify that $\alpha$ and $U$ fulfil property (2.1) of Lemma 2.1.

The authors of [1] use families of trees $\left(T_{a},<_{a}\right)_{a \in A}$ satisfying $(* *)$ of Example 3.5 to produce several examples of compact spaces $\Omega$ in this way, including a non-Gul'ko space. Every tree considered in [1 has height $\omega$, so is certainly special. If each $\left(T_{a},<_{a}\right)$ is special then none contain uncountable intervals, so the corresponding compact space $\Omega$ is Corson. From above, it follows that $\Omega$ is also descriptive. In particular, we have another example of a Corson, descriptive, non-Gul'ko space.

\section{REFERENCES}

1. S. Argyros, A. Arvanitakis and S. Mercourakis, Reznichenko families of trees and their applications. Forthcoming in J. Math. Anal. Appl.

2. S. Argyros, S. Mercourakis and S. Negrepontis, Functional-analytic properties of Corsoncompact spaces. Studia Math. 89 (1988), 197-229.

3. S. Argyros and S. Mercourakis, On weakly Lindelöf Banach spaces. Rocky Mountain J. Math. 23 (1993), 395-446.

4. M. Day, Strict convexity and smoothness of normed spaces. Trans. Amer. Math. Soc. 78 (1955), 516-528.

5. R. Deville, G. Godefroy and V. Zizler, Smoothness and Renormings in Banach Spaces. (Longman, Harlow, 1993). 
6. M. Fabian, Gâteaux Differentiability of Convex Functions and Topology. (John Wiley and Sons, Inc., New York, 1997).

7. G. Gruenhage, Generalized metric spaces, in K. Kunen and J. E. Vaughan, editors, Handbook of set-theoretic topology, 235-293, North Holland, Amsterdam, 1984.

8. G. Gruenhage, A note on Gul'ko compact spaces. Proc. Amer. Math. Soc. 100 (1987), 371-376.

9. R. W. Hansell, Descriptive sets and the topology of nonseparable Banach spaces. Serdica Math. J. 27 (2001), 1-66.

10. J. Lindenstrauss and L. Tzafriri, Classical Banach Spaces I. (Springer-Verlag, Berlin-New York, 1977).

11. A. G. Leiderman and G. A. Sokolov, Adequate families of sets and Corson compacts Comment. Math. Univ. Carolin. 25 (1984), 233-246.

12. A. Moltó, J. Orihuela, S. Troyanski and V. Zizler, Strictly convex renormings. J. London Math. Soc. 75 (2007), 647-658.

13. L. Oncina and M. Raja, Descriptive compact spaces and renorming. Studia Math. 165 (2004), 39-52.

14. M. Raja, Weak* locally uniformly rotund norms and descriptive compact spaces. J. Funct. Anal. 197 (2003), 1-13.

15. N. K. Ribarska, Internal characterization of fragmentable spaces. Mathematika 34 (1987), 243-257.

16. R. J. Smith, Gruenhage compacta and strictly convex dual norms. J. Math. Anal. Appl. 350 (2009), 745-757.

17. M. Talagrand, Espaces de Banach faiblement $\mathscr{K}$-analytiques Ann. of Math. 110 (1979), 407438.

18. S. Troyanski, Equivalent norms in nonseparable B-spaces with an unconditional basis, Teoria funkcii, Funkcional. Anal. i Prilozhenia 6 (1968), 92-96 (Russian).

Institute of Mathematics of the AS CR, Žitná 25, CZ - 11567 Praha 1, Czech REPUBLIC

E-mail address: smith@math.cas.cz

Departamento de Matemticas, Universidad de Murcia, Campus de Espinardo., 30100 Murcia, SPAin

E-mail address: stroya@um.es 